\title{
Prevalence of Methicillin Resistant Staphylococcus aureus Post Operative Wound Infections in a Trust Hospital
}

\author{
D. Neelaveni ${ }^{1^{*}}$ and S. Ramasamy ${ }^{2}$ \\ ${ }^{1}$ Department of Microbiology, Government Mohan Kumaramangalam Medical \\ College, Salem, India \\ ${ }^{2}$ Department of Medicine, Government Mohan Kumaramangalam Medical College, Salem, India \\ *Corresponding author
}

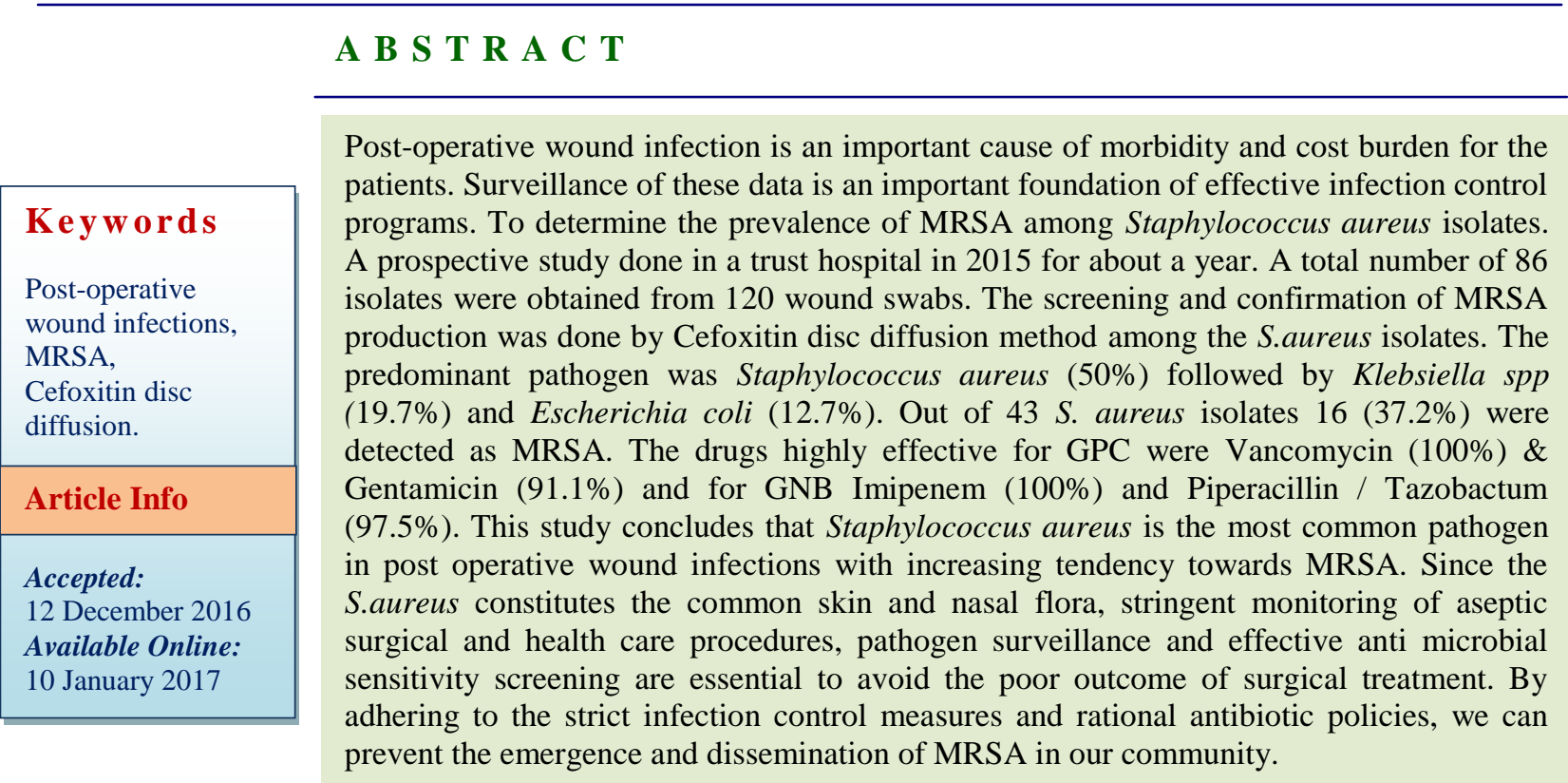

\section{Introduction}

Post operative wound infections (POWI) or surgical site infections (SSI) are the most common Procedure and Health care associated nosocomial infections (14 \%-16\%) (Centers for Disease Control and Prevention, 2007). SSIs are identified in surgical ward patients mostly associated with Cardiac, Abdominal, Orthopedics and Neurosurgery procedures (Ibtesam et al., 2010). Postoperative wound infections can be caused by two major sources: exogenous and endogenous bacteria (Amrita et al., 2010). The most common predisposing factors for these infections are prolonged hospital stay, increased duration of surgery, extensive handling of tissues during procedures, impaired immune status of the patient, nutritional deficiency. These factors will lead to increased morbidity and mortality in postoperative patients. Micro-organisms responsible for wound infection depends on the surgical site, patient's systemic host defenses, local wound conditions, microbial burden and antimicrobial use in the hospital. The outcome of antimicrobial therapy, both prophylactically and therapeutically can only 
be defined when these factors are under control. These infection rates are an established measure of quality of clinical care and provision of reliable surveillance data is the foundation for effective infection control programs (Khaleid et al., 2010).

Antimicrobial-resistant pathogens that cause healthcare-associated infections pose an increasing challenge to hospitals, both in the clinical treatment of patients and in the prevention of the cross-transmission of this problematic pathogens (Khaleid et al., 2010). Since the regular surveillance could play a significant role in the early recognition \& intervention for better management, the study was designed to evaluate the prevalence of bacteriological profile in surgical site infections.

The main of this study includes, to detect the bacterial profile, to evaluate their antimicrobial sensitivity pattern in post operative wound infections and to identify the MRSA among Staphylococcus aureus isolates.

\section{Materials and Methods}

A prospective study was conducted during the period from December 2014- November 2015 in a trust hospital, shevapet, Salem, after obtaining informed consent from patients admitted in post operative wards with complaints of wound discharge and pain in the operated site. The samples were collected from total no of 120 patients, two samples (swabs) from each were collected and transported to the Microbiology laboratory immediately and processed as per standard guidelines.

\section{Bacterial isolation procedure}

The first swab was used for direct Gram's staining and the smear was observed for pus cells \& bacteria under oil immersion (100x).
The second swab was inoculated immediately into the following media for bacterial isolation namely, Nutrient agar, MacConkey agar and Blood agar. Streak culture method was followed for inoculation, and the plates were incubated at $37 \mathrm{C}$ for 24 hours. After incubation, the pure isolates were observed and identified by their colony morphology which were then confirmed \& speciated as per standard biochemical reactions (Washington et al., 2006).

Antimicrobial sensitivity testing was done for all bacterial isolates as per Clinical and Laboratory Standards Institute (CLSI) guidelines by Disc Diffusion method. The Gram positive cocci were tested against the panel of antibiotic discs like Ampicillin (10 $\mu \mathrm{g})$, Erythromycin $(15 \mu \mathrm{g})$, Amox/clav (20/10 $\mu \mathrm{g})$, Cotrimoxazole (23.75/ $1.25 \quad \mu \mathrm{g})$, Gentamicin $(10 \mu \mathrm{g})$, Ciprofloxacin $(5 \mu \mathrm{g})$ and Vancomycin $(30 \mu \mathrm{g})$. Along with the above discs for screening \& confirmation of Methicillin resistance Cefoxitin $(30 \mu \mathrm{g})$ discs were used ${ }^{7}$ and Quality control strains, MRSA ATCC 43300 and Methicillin-sensitive $S$. aureus (MSSA) ATCC 25923, were used as positive and negative controls respectively (Clinical and Laboratory Standards Institute, 2013; Bhadravathi et al., 2014).

The Gram negative bacilli were tested against the panel of antibiotic discs namely, Cotrimoxazole $(23.75 / 1.25 \mu \mathrm{g})$, Amikacin (30 $\mu \mathrm{g})$ Gentamicin $(10 \mu \mathrm{g})$, Ciprofloxacin $(5 \mu \mathrm{g})$, Cefotaxime $(30 \mu \mathrm{g})$, Ceftazidime $(30 \mu \mathrm{g})$, Amox/clav $(20 / 10 \mu \mathrm{g})$, Imipenem $(10 \mu \mathrm{g})$ and Piperacillin / Tazobactum $(100 / 10 \mu \mathrm{g})$. The results were recorded and interpreted as per the recommendations of the Clinical and Laboratory Standards Institute (CLSI).

\section{Results and Discussion}

Out of total population (120), 73 male $(60.8 \%)$ and 47 females $(39.1 \%)$ were affected by post operative wound infection. From total 
samples, 86 were positive for culture which includes 52 male $(60.4 \%)$ and 34 females $(39.5 \%)$. The samples collected from General surgery and OG departments showed culture positivity in $61(70.9 \%$ ) and $25(29 \%)$ samples respectively.

Among the 86 culture positive specimens, 45 Gram Positive Cocci (52.3\%) and 41Gram Negative Bacilli (47.6\%) were isolated. Among the isolates, Staphylococcus aureus $(50 \%)$ was the predominant isolate, followed by Klebsiella spp (19.7\%), Escherichia coli (12.7\%), Proteus spp (8.1\%), Pseudomonas aeruginosa (6.9\%) and the least is CONS $(2.3 \%)$ [Chart -1].

The Antibiotic sensitivity pattern of Gram positive cocci showed sensitivity to Ampicillin (33.3\%), Erythromycin (28.8\%), Amox/clav (55.5\%), Cotrimoxazole (68.8\%), Gentamicin (91.1\%), and Ciprofloxacin $(75.5 \%)$ and Vancomycin (100\%). Among 43 Staph aureus isolates $16(37.2 \%)$ were identified and confirmed phenotypically as MRSA by Cefoxitin disc diffusion method. The results with zone inhibition diameter for cefoxitin $(30 \mu \mathrm{g}$ per disc $) \leq 21 \mathrm{~mm}$ indicated resistance to Methicillin [Table -1].

The antibiotic sensitivity pattern of Gram Negative Bacilli showed sensitivity to Cotrimoxazole (34.1\%), Amikacin (87.8\%), Gentamicin (85.3\%), Amox/clav (51.2\%), Ciprofloxacin (65.8\%), Cefotaxime (90.2\%), Ceftazidime (46.3\%), Imipenem (100\%) and Piperacillin /Tazobactum $(97.5 \%)$ [Table - 2].

The incidence of post operative wound infections is increasing in alarming rate and has been a problem in the field of surgery for a long time. Most of the organisms occur as saprophytes and colonise the skin, nasopharynx and gastro-intestinal tract with little potential for causing disease because they induce first line defense mechanisms inside the body. Skin barrier is disrupted by surgical procedures, diseases, nutritional deficiency and other factors that affect the first line defenses.

The present study showed that male patients (60.4\%) were affected more than females $(39.5 \%)$, and the most affected age group was $>50$ years of age. which is similar to the study by Amrita et al., (male $72 \%$ and females 28\%) and Adegoke et al., This may be due to the increased prevalence of other illnesses like diabetes, malnutrition etc and increased chances of injury for male patients by occupation, and they are more prone to acute abdomen problems than females

In this present study S.aureus $(50 \%)$ was the predominant isolate in postoperative wound infection. [Table 1] This is supported by various studies like Amrita et al study which showed it to be $63 \%$, other studies by Khaleid et al., showed 42.6\%, Arti et al., as $32 \%$, and Adegoke et al., as 25\% (Afrough et al., 2012; Sarita yadav et al., 2010).

The present study showed, the range of Gram negative isolates include Klebsiella spp (19.7 $\%)$, Escherichia coli (12.7\%), Proteus spp (8.1\%) and Pseudomonas aeruginosa (6.6\%). This result is supported by Amrita et al's study showing the Gram negative isolates in post operative wound infections namely Escherichia coli $9.5 \%$, Pseudomonas spp 5 $\%$, Klebsiella spp $3.5 \%$ and Proteus spp $3.5 \%$. Khaleid M. et al study showed that Klebsiella spp, P. aeruginosa and E. coli were detected in the frequency of $19.2 \%, 10.6 \%$ \& $4.3 \%$ respectively and also in Maida Sisirak et al., study Escherichia coli $8.7 \%$, Pseudomonas spp $8.9 \%$, Klebsiella spp 3.5\% and Proteus spp $6.5 \%$ were isolated in surgical site infections.

Antimicrobial sensitivity of Gram positive cocci showed highest sensitivity for Vancomycin $100 \%$. The lowest sensitivity pattern was towards Ampicillin 33.3\% and 
Erythromycin $28.8 \%$ [Table 2]. This report is similar to Amrita et al., and Adegoke et al., studies which showed sensitivity to Vancomycin 100\%, Ampicillin 1.8\% \& 15\%, and Gentamicin $98 \% \& 20 \%$ respectively.

In the present study, sensitivity pattern of Gram Negative Bacilli showed highest sensitivity towards Imipenem 100\%, Piperacillin/ Tazobactum 97.5\%, and other third generation Cephalosporins $60-90 \%$ and Amino glycoside 86\% \& Quinolones 60-70\% respectively. The lowest sensitivity was towards Cotrimoxazole $34.1 \%$ [Table 3]. This is similar to the study by Amrita et al., which showed sensitivity to Aminoglycosides, Quinolones and Cephalosporins in the range of $40-60 \%$.

In this study, 20 MRSA strains (46.5\%) were isolated out of 43 Staph aureus isolates [Table $2]$. This report is similar to other studies by Khaleid et al., (65\%), Sarita et al., (60.6\%), Bhutia et al., (42.1\%) and Pillai et al., (53.4\%) with MRSA isolates.

In conclusion, the Centre for Disease Control and Prevention (CDC) in Atlanta, USA, estimates that $2.7 \%$ of surgical procedures are complicated by infections. Surgical site infections (SSI) represent 16\% of all hospitalacquired infections, making them the third most frequent type of nosocomial infections in developed countries. These infections increase the mortality and morbidity, prolong hospitalization and have adverse impact on clinical outcome (Kirkland et al., 1999). It is very useful to explore the causative agents of these infections to demonstrate the magnitude of the problem.

Table.1 Antimicrobial sensitivity pattern for GPC (number and percentage)

\begin{tabular}{|c|l|c|c|c|}
\hline S.No & Name of the Drugs & Staph. aureus $(\mathrm{N}=43)$ & CONS(N=2) & $\begin{array}{l}\text { Total no of } \\
\text { Isolates (N=45) }\end{array}$ \\
\hline 1. & Ampicillin (A) & $15(34.8 \%)$ & $0(0 \%)$ & $15(33.3 \%)$ \\
\hline 2. & Erythromycin (E) & $13(30.2 \%)$ & $0(0 \%)$ & $13(28.8 \%)$ \\
\hline 3. & Amox/clav (AMC) & $24(55.8 \%)$ & $1(50 \%)$ & $25(55.5 \%)$ \\
\hline 4. & Cotrimoxazole (CO) & $30(69.7 \%)$ & $1(50 \%)$ & $31(68.8 \%)$ \\
\hline 5. & Gentamicin (G) & $39(90.6 \%)$ & $2(100 \%)$ & $41(91.1 \%)$ \\
\hline 6. & Ciprofloxacin(CF) & $31(72 \%)$ & $2(100 \%)$ & $34(75.5 \%)$ \\
\hline 7. & Vancomycin (V) & $43(100 \%)$ & $2(100 \%)$ & $45(100 \%)$ \\
\hline 8. & Cefoxitin (CX) & $16(37.2 \%)$ & $2(100 \%)$ & $18(40 \%)$ \\
\hline
\end{tabular}

Table.2 Antimicrobial sensitivity pattern for GNB (number and percentage)

\begin{tabular}{|c|l|l|l|l|l|l|}
\hline S.No & Name of the Drugs & $\begin{array}{l}\text { Klebsiella } \\
\mathrm{spp}(\mathrm{n}=17)\end{array}$ & $\begin{array}{l}\text { E.coli } \\
(\mathrm{n}-11)\end{array}$ & $\begin{array}{l}\text { Proteus spp } \\
(\mathrm{n}=7)\end{array}$ & $\begin{array}{l}\text { P. aeruginosa } \\
(\mathrm{n}=6)\end{array}$ & $\begin{array}{l}\text { Total no of } \\
\text { isolates }(\mathrm{n}-41)\end{array}$ \\
\hline 1 & Cotrimoxazole (CO) & $6(35.2 \%)$ & $5(45.4 \%)$ & $3(42.8 \%)$ & $0(0 \%)$ & $14(34.1 \%)$ \\
\hline 2 & Amikacin (AK) & $16(94.1 \%)$ & $10(90.9 \%)$ & $5(71.4 \%)$ & $5(83.3 \%)$ & $36(87.8 \%)$ \\
\hline 3 & Gentamicin (G) & $15(88.2 \%)$ & $10(90.9 \%)$ & $5(71.4 \%)$ & $5(83.3 \%)$ & $35(85.3 \%)$ \\
\hline 4 & Ciprofloxacin (CF) & $12(70.5 \%)$ & $8(72.7 \%)$ & $4(57.1 \%)$ & $3(50 \%)$ & $27(65.8 \%)$ \\
\hline 5 & Cefotaxime (CE) & $15(88.2 \%)$ & $10(90.9 \%)$ & $5(71.4 \%)$ & $2(33.3 \%)$ & $37(90.2 \%)$ \\
\hline 6 & Ceftazidime(CAZ) & $10(58.8 \%)$ & $5(45.4 \%)$ & $2(28.5 \%)$ & $2(33.3 \%)$ & $19(46.3 \%)$ \\
\hline 7 & Imipenem(IMP) & $17(100 \%)$ & $11(100 \%)$ & $7(100 \%)$ & $6(100 \%)$ & $41(100 \%)$ \\
\hline 8 & Pip / Taz (PIT) & $17(100 \%)$ & $10(90.9 \%)$ & $7(100 \%)$ & $6(100 \%)$ & $40(97.5 \%)$ \\
\hline 9 & Amox/Clav (AMC) & $9(52.9 \%)$ & $8(72.7 \%)$ & $3(42.8 \%)$ & $1(16.6 \%)$ & $21(51.2 \%)$ \\
\hline
\end{tabular}




\section{Chart .1}

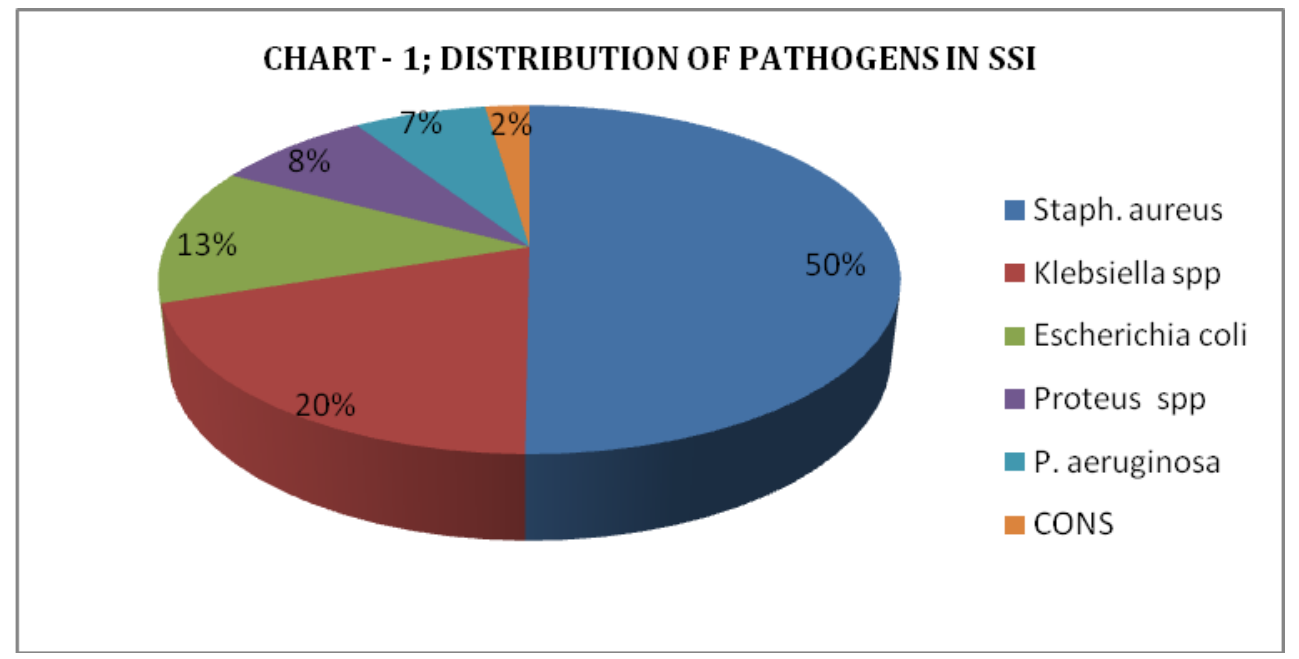

In this study among total 120 samples, 86 were culture positive. Out of them $(60.4 \%)$ were male and 44(39.5\%) were females. Predominant isolate among total organisms 43 were S.aureus. Among the S.aureus isolates, $20(46.5 \%)$ MRSA were detected by Cefoxitin disc diffusion method. All MRSA isolates were sensitive to Vancomycin.

The study showed Staphylococcus aureus as the major pathogen in post operative wound infections and increasing incidence of MRSA in wound infection. Since the patients colonized with MRSA have an increased risk for developing infection compared with noncolonized patients and it can be easily transmitted to inpatients through health workers and patient attenders. Therefore, rapid and accurate identification of multi-drug resistant Staphylococcus aureus is crucial and essential both for initiation of appropriate antimicrobial therapies and for effective infection control strategies to limit the spread of MRSA. In spite of the fact that there are many new rapid and less costly methodologies, the traditional susceptibility testing, such as disk diffusion method with cefoxitin, is available in every bacteriological laboratory and this method can be performed easily to detect MRSA (Mohammed Reza et al., Gargi Dangre et al., \& Shadi et al.,). It also remains the most recommended methods of CLSI. The present study showed that $S$. aureus isolates were also resistant to other common \& routinely used antibiotics like Erythromycin and Cotrimoxazole. A general overview of the antibiogram of all the bacterial isolates indicated that Gram positive bacteria exhibited a greater level of antimicrobial sensitivity to Vancomycin $(100 \%)$ and Gram negative bacteria to Imipenem (100\%) \& Piperacillin/ Tazobactum $(97.5 \%)$ So it is the need of the hour to evaluate the infection control measures such as the proper pathogen surveillance, antimicrobial sensitivity pattern and enforcement of hand washing by health workers and these may help in arresting the spread of MRSA and other resistant pathogens in hospital settings. An antibiotic policy and the monitoring of sensitivity patterns for pathogens may also help in decreasing the prevalence of MRSA and antibiotic resistance, thereby preventing the morbidity and mortality in postoperative patients.

\section{References}

Adegoke, Anthony, A., Tom Mvuyo, Okoh, Anthony, I. and Jacob Steve. 2010. 
Studies on multiple antibiotic resistant bacteria isolated from surgical site infection; Academic J. Scientific Res. Essays, Vol. 5(24), p. 3876-3881.

Afrough, P., Pourmand, M.R., Zeinalinia, N., Yousefi, M., Abdossamadi, Z., Yazdchi, S. 2012. Molecular typing of clinical and nasal carriage isolates of staphylococcus aureus by spa gene patterns. J. Maz. Univ. Med., 22: 28-34.

Amrita shriyan, Sheetal, R. and Narendra Nayak. 2010. Aerobic Micro organisms in post operative wound infections and their antimicrobial susceptibility patterns; J. clin. Diag. res., Vol.4(6), p. $3392-3396$

Betty, A., Forbes, Daniel, F., Sahm and Alice S., Weissfeld-Bailey \& Scott's Diagnostic Khaleid, M., Abdel-Haleim, Zeinab AbdelMicrobiology; XII edition, Mosby, 2007, p. 80-82, 187-197.

Bhadravathi Virupaksha Renushri, Avinandan Saha, Elkal Rajappa Nagaraj, N.K. Rama, Veena Krishnamurthy, SC Chandrashekar. 2014. Screening for methicillin-resistant Staphylococcus aureus carriers among individuals exposed and not exposed to the hospital environment and their antimicrobial sensitivity pattern, Annal of Tropical Medicine and Public Health, Volume: 7, Issue : 1, Page : 19-24.

Bhutia, K.O., Singh, T.S., Biswas, S., Adhikari, L. 2012. Evaluation of phenotypic with genotypic methods for species identification and detection of methicillin resistant in Staphylococcus aureus. Int. J. Appl. Basic Med. Res., 2: 84-91.

Centers for Disease Control and Prevention (CDC). 2007. The National Healthcare Safety Network (NHSN) Manual. Patient Safety Component protocol. Division of Healthcare Quality Promotion; Available at: http//www. cdc.gov/ncidod/dhqp/pdf/nhsn
Clinical and Laboratory Standards Institute. 23rd informational supplement M100-S23. CLSI; Wayne, PA: 2013. Performance standards for antimicrobial susceptibility testing

Gargi Dangre-Mudey, Abhay Mudey, Neelima Tankhiwale. 2015. Study of nasal carriage of Staphylococcus aureus among health care workers of a rural tertiary health care centre, International $J$. Healthcare and Biomed. Res.., Volume: 03, Issue: 03, Pages 85-90.

Ibtesam, K., Afifi and Ehssan, A., Baghagho. 2010. Three months study of Orthopedic Surgical Site infections in an Egyptian University hospital, Int. $J$. Infect. Control, vol 6: i - 1. Khalek Ibraheim and Eman M. ElTahlawy. 2010. Surgical Site Infections and Associated Risk Factors in Egyptian Orthopedic Patients; $J$. American Sci., Vol.6 (7),p.272-280

Mediavilla, J.R., Chen, L., Mathema, B., Kreiswirth, B.N. 2012. Global epidemiology of community-associated methicillin resistant Staphylococcus aureus (CA-MRSA) Curr. Opin. Microbial., 15: 588-595.

Mohammad Reza Pourmand, Sepideh Hassanzadeh, Rahil Mashhadi and Emran Askari. Comparison of four diagnostic methods for detection of methicillin resistant Staphylococcus aureus Iran J. Microbiol., 6(5): 341-344

Pillai, M.M., Latha, R., Sarkar, G. 2012. Detection of methicillin resistance in Staphylococcus aureus by polymerase chain reaction and conventional methods: A comparative study. J. Lab Physicians, 4: 83-88.

Sarita yadav, Aparna yadav, Madhu sharma and Uma Chaudhary. 2010. Prevalence and sensitivity pattern of Staphylococcus 
aureus in surgical wound infections; Int. J. Pharma and Bio sci., vol.1(3), p.1-3.

Shadi, A., Zakai. 2015. Prevalence of methicillin-resistant Staphylococcus aureus nasal colonization among medical students in Jeddah, Saudi Arabia, Saudi Med. J., 36(7): 807-812.
Washington winn, Jr. Stephen Allen, William Janda, Elmer Koneman, Gary Procop and Paul Schreckenberger et al - Kone man's Color Atlas and Text book of Diagnostic Microbiology; Sixth edition, Lippincott Williams \&Wilkins, p. 945 -1015.

\section{How to cite this article:}

Neelaveni, D., and Ramasamy, S. 2017. Prevalence of Methicillin Resistant Staphylococcus aureus Post Operative Wound Infections in a Trust Hospital. Int.J.Curr.Microbiol.App.Sci. 6(1): 251-257. doi: http://dx.doi.org/10.20546/ijcmas.2017.601.030 